\title{
Tree Shape Priors with Connectivity Constraints using Convex Relaxation on General Graphs
}

\author{
Jan Stühmer \\ TU München, Caltech
}

\author{
Peter Schröder \\ Caltech
}

\author{
Daniel Cremers \\ TU München
}

\begin{abstract}
We propose a novel method to include a connectivity prior into image segmentation that is based on a binary labeling of a directed graph, in this case a geodesic shortest path tree. Specifically we make two contributions: First, we construct a geodesic shortest path tree with a distance measure that is related to the image data and the bending energy of each path in the tree. Second, we include a connectivity prior in our segmentation model, that allows to segment not only a single elongated structure, but instead a whole connected branching tree. Because both our segmentation model and the connectivity constraint are convex, a global optimal solution can be found. To this end, we generalize a recent primal-dual algorithm for continuous convex optimization to an arbitrary graph structure. To validate our method we present results on data from medical imaging in angiography and retinal blood vessel segmentation.
\end{abstract}

\section{Introduction}

The task of image segmentation, the separation of an image into meaningful parts, is one of the most important and well studied problems in image processing and computer vision. While state-of-the-art segmentation methods [8, 18, 29] perform well for segmenting compact objects, their performance on thin and elongated structures is often not satisfying. The commonly used length regularizer suppresses small structures and the correct topology cannot be reconstructed.

To overcome this shrinking bias, recently two different approaches have been suggested in the literature. First, curvature based measures have attracted the interest of researchers in computer vision to include them in image segmentation frameworks $[17,25,14]$. However, introducing these regularizers into segmentation algorithms leads to higher order cost functions, which are hard to optimize.

Another way to preserve thin structures is to use topological constraints. A special subclass of these constraints are connectivity constraints, which ensure the connectedness of a labeled region and therefore allow that thin connections

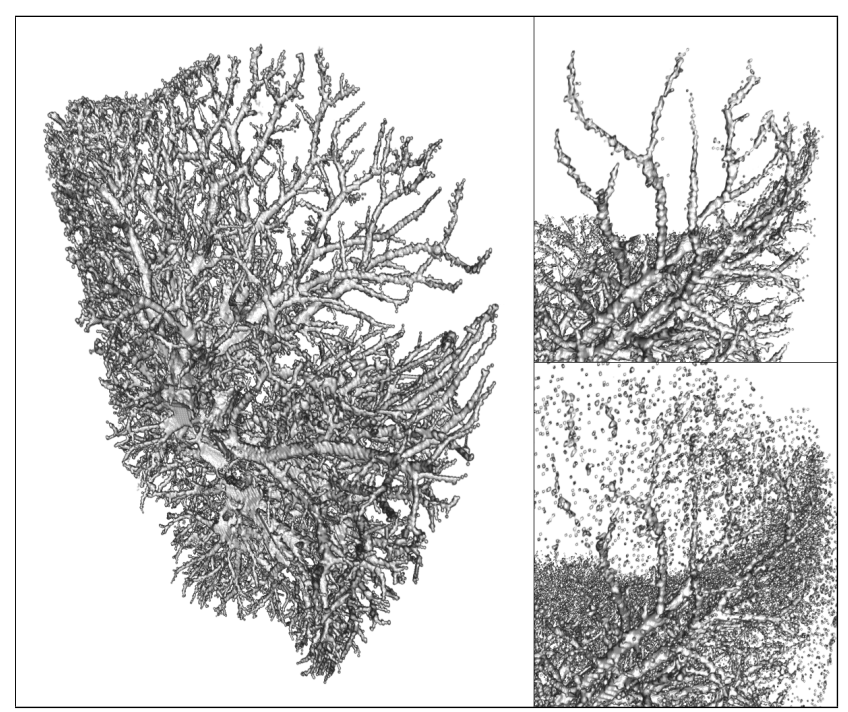

Figure 1: Segmentation of a blood vessel tree of the lung in angiography. The connectivity constraint allows to connect previously unconnected regions while noise is successfully removed. Left image and top right: Lung vessel tree segmented with the proposed method. Bottom right: Result of the algorithm using the same parameters but without the connectivity constraint.

between foreground regions are preserved in the final segmentation result. To overcome the limitation of topology preserving level set methods [19], that only locally optimal solutions can be achieved, recent approaches include topological constraints in random field models [30, 23, 11]. So far, these methods only allow to compute an approximate solution of the global optimization problem.

\subsection{Related Work}

In Zeng et al. [31] a topology preserving extension of the GraphCut method [9] is presented. The method is initialized on a coarser scale and then refines the segmentation while preserving the topology. Similar to topology preserving level set methods [19] this method converges only to a local minimum of the energy. 
Vicente et al. [30] introduce a Markov random field segmentation model extended with a connectivity constraint for user given seed nodes, that should be connected to the foreground segment. They prove that even when the model only contains unary terms solving the image segmentation problem with these constraints is NP-hard. Therefore they introduce a heuristic algorithm that is essentially a Dijkstra algorithm into which a graph cut algorithm is embedded, but which can only provide an approximate solution when the random field contains pairwise energies.

Another recent approach to enforce connectivity of the foreground region is the work of Nowozin and Lampert [23]. They formulate the constrained image segmentation problem as a linear programming (LP) relaxation which can in principle be solved globally optimally. Because the relaxation is not tight their algorithm is not guaranteed to converge to a binary solution. Additionally, the LP-relaxation does not scale well with the image size, limiting the method to a problem size of a few hundred pixels.

One application field of methods that preserve elongated structures is in angiography, were thin structures like blood vessels need to be segmented. Some of the most promising existing methods for this special task are based on geodesic shortest paths. By using a local anisotropic metric and modeling the segmentation task as a path search problem with varying radius, of circles for $2 \mathrm{D}$ images [5] and spheres for 3D data [4], such methods are well suited for the special case of tubular structures like blood vessels, but at the same time are restricted to this specific task. Instead of modeling the objects that should be segmented explicitly as connected paths some authors propose to first preprocess the image data with filters that show a strong response in areas were elongated structures are present $[16,21]$. In the recent work of Bauer et al. [3] a similar approach leads to an explicit model of short tubular segments that are in a second step connected to a whole tree of branching tubular structures. Therefore a connection confidence measure to join adjacent tube segments is defined, that depends on the distance and joining angle of the segments. The resulting minimization problem is solved by using the graph cut algorithm [9]. For a review on recent work in the particular application domain of blood vessel segmentation see [22].

\subsection{Problem Formulation}

Given an image $I$ with the domain $\Omega$, a bounded connected subset of $\mathbb{R}^{m}$, we wish to solve the constrained optimization problem

$$
\begin{aligned}
& \min _{\ell \in\{0,1\}} \int_{\Omega} f(x) \ell(x) d x+\lambda \operatorname{Per}\left(\Sigma_{\ell=1}\right) \\
& \text { s.t. } \\
& \forall x, x^{\prime} \in \Sigma_{\ell=1}: \exists C_{x}^{x^{\prime}} \subset \Sigma_{\ell=1} .
\end{aligned}
$$

The discrete label assignment $\ell: \Omega \rightarrow\{0,1\}$ describes if an image region belongs to the object of interest $\ell(x)=1$ or the image background $\ell(x)=0$. The set $\Sigma_{\ell=1}=$ $\{x \in \Omega: \ell(x)=1\}$ is the foreground region, a subset of the image domain. With $C_{x}^{x^{\prime}}$ we formalize a connected trajectory from $x$ to $x^{\prime}$ as a continuous function $C_{x}^{x^{\prime}}:[0, T] \rightarrow \Omega$ with $C_{x}^{x^{\prime}}(0)=x$ and $C_{x}^{x^{\prime}}(T)=x^{\prime}$. The function $f(x)=\log \frac{P(I(x) \mid \ell(x)=0)}{P(I(x) \mid \ell(x)=1)}$ is a probabilistic model that depends on the image data.

The solution of the optimization problem should satisfy the connectivity constraint $\mathrm{C} 0$ :

For each $x, x^{\prime} \in \Omega$ that belong to the foreground there must exist a connected path from $x$ to $x^{\prime}$ such that all $p \in \Omega$ in the path between $x$ and $x^{\prime}$ belong to the foreground.

This constraint ensures that the whole foreground region is connected. Unfortunately even for the special case $\lambda=0$, minimizing Eq. 1 with $\mathrm{C} 0$ is NP-hard because the minimum Steiner tree problem can be reduced to this problem [30].

\subsection{Contribution}

In this work, we propose to reformulate the connectivity constraint on the fixed topology of a discrete graph that is constructed from a shortest geodesic path tree. While solving the original problem is NP-hard, the reformulated problem can be solved to global optimality in polynomial time. We show how a-priori information about the geometry of the structure of interest can be included when constructing this shortest path tree. To solve the resulting labeling problem, we generalize a recent primal-dual algorithm for continuous convex optimization to an arbitrary graph.

\section{Approximation with Shortest Paths Trees}

To approximate the solution to Eq. 1 we propose to reformulate the connectivity constraint on a fixed topology. We choose the shortest geodesic path tree $\mathcal{G}_{s}$ originating in a given $s \in \Omega$ that contains the shortest path to every other position in the image. Our reformulated connectivity constraint $\mathrm{C} 1$ can be formalized as

$$
\forall x \in \Omega, \ell(x)=1: \exists C_{s}^{x} \in \mathcal{G}_{s}: \ell\left(C_{s}^{x}(t)\right)=1 .
$$

In contrast to the original image segmentation problem, we will show in section 3.3 that this constraint can be introduced as a linear constraint in a convex optimization framework and therefore allows for an optimal solution.

\subsection{Constructing the Geodesic Shortest Path Tree}

The shortest geodesic path topology is inspired by image segmentation methods based on shortest geodesic distances, that have been successfully applied to medical image segmentation [4] and general image segmentation as well $[2,12]$. It allows to use an image depending local geodesic metric and additionally to incorporate a-priori knowledge about the geometry of the object of interest. 
First we have to choose an appropriate local geodesic metric. If $\lambda=0$ the labeling function $u(x)$ takes the value 1 for $f(x)<0$ and 0 for $f(x)>0$. We leave out the special case $f(x)=0$ as it does not occur in practice. For all $x_{p} \in \Omega$ that do not belong to the foreground but need to be added to the foreground to satisfy the connectivity constraint obviously $u\left(x_{p}\right)=0$ and therefore $f\left(x_{p}\right) \geq 0$. The optimal cost of the connecting path between a fixed $s$ and any $x$ in the region that should be connected on $\mathcal{G}_{s}$ is then given by

$$
\min _{C_{s}^{x}} \int_{0}^{T} f^{+}(C(t)) d t
$$

with $f^{+}=\max (0, f(x))$. Thus, we choose the non negative cost function $f^{+}$as metric for the construction of $\mathcal{G}_{s}$.

\subsection{Bending Energy Prior}

Further a-priori information about the geometry of the object that should be segmented can be included in the framework. For the special case of blood vessel segmentation in medical imaging a reasonable assumption would be, that the structure of interest ideally minimizes its bending energy. The probability of a discretized position to reside inside the structure can be modeled as

$$
P\left(x_{t}\right) \sim \exp \left\{-E_{\text {bend }}\left(x_{t}\right)\right\}
$$

where $E_{\text {bend }}\left(x_{t}\right)$ is the local part of the bending energy that would add to the total bending energy of a curve, if it was going through $x_{t}$ given the positions of its neighboring nodes $x_{t-1}$ and $x_{t+1}$.

To compute the bending energy we use the discretized bending energy of Bergou et al. [6] that can be applied for curves in 2D as well as spacecurves in 3D. It is expressed using the curvature binormal

$$
(\kappa \mathbf{b})_{t}=\frac{2 \mathbf{e}^{t-1} \times \mathbf{e}^{t}}{\left|\mathbf{e}^{t-1}\right|\left|\mathbf{e}^{t}\right|+\mathbf{e}^{t-1} \cdot \mathbf{e}^{t}}
$$

with $e^{t}=x_{t+1}-x_{t}$ and $e^{t-1}=x_{t}-x_{t-1}$. Because this is an integrated quantity, dividing it by the length of the domain of integration, in this case half the length $l_{t}=$ $\left|e^{t}\right|+\left|e^{t-1}\right|$ of the edges joining at $x_{t}$, gives the discretized version of the bending energy

$$
E_{\mathrm{bend}}\left(x_{t}\right)=\frac{1}{2} \alpha\left(\frac{(\kappa \mathbf{b})_{t}}{l_{t} / 2}\right)^{2} \frac{l_{t}}{2}=\frac{\alpha(\kappa \mathbf{b})_{t}^{2}}{l_{t}} .
$$

The curvature binormal $(\kappa \mathbf{b})_{t}$ depends not only on the position $x_{t}$ but also on the positions of the neighboring nodes $x_{t+1}$ and $x_{t-1}$.

Finally, the combination of the non negative data term and the bending energy prior lead to the shortest path problem

$$
\min _{C} \int_{0}^{T} f^{+}(C(t))+E_{\text {bend }}(C(t)) d t .
$$
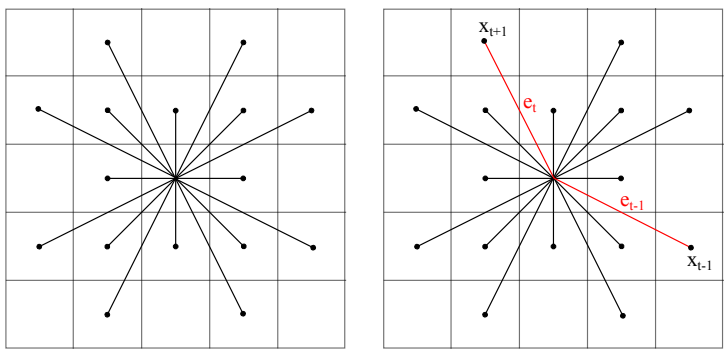

Figure 2: Discretized neighborhood on the pixel grid that is used for the shortest path search. The bending energy depends on the two edges $e_{t-1}$ and $e_{t}$ joining at a node $x_{t}$.

Note that this is not a usual geodesic measure, because the bending energy term depends on the incoming and outgoing angle. Thus, standard first-order techniques [28, 26] cannot be used. Instead, such cost functions can be minimized by computing a shortest path on a higher order graph, which contains a node for every edge in the original graph i.e. for every pair of nodes that are connected by an edge in the original graph [1]. Because our major contribution in this paper is the labeling of the nodes via convex optimization, we approximate the minimal path using a greedy optimization scheme, in this case Dijkstra's [13] algorithm on the vertex graph. At every expansion step of Dijkstra's algorithm the value of Eq. 6 for the candidate nodes $x_{t+1}$ is computed by taking the predecessor $x_{t-1}$ in the current shortest path to $x_{t}$. Thus this approximation does not take into account different incoming angles to the node $x_{t}$ but assumes this angle to be fixed by $x_{t-1}$.

\section{Labeling the Shortest Geodesic Path Tree via Convex Optimization}

To formulate Eq. 1 in a convex optimization framework the discrete label assignment $\ell: \Omega \rightarrow\{0,1\}$ is relaxed by introducing a continuous indicator function $u: \Omega \rightarrow$ $[0,1]$. The boundary length of the foreground segment can be measured by using the total variation of $u$, which is a convex function.

In the continuous framework, the image segmentation problem with $\mathrm{C} 1$ has the form

$$
\min _{u \in[0,1]} \int_{\Omega} f(x) u(x)+\lambda|\nabla u| d x
$$

s.t.

$\forall x \in \Omega, u(x)=1: \exists C_{s}^{x} \in \mathcal{G}_{s}: u\left(C_{s}^{x}(t)\right)=1$.

In the following we show how this continuous image segmentation problem can be equivalently transformed to the domain of a discrete graph. We define the corresponding operators on a weighted graph and derive a local varia- 
tion regularized segmentation model as theoretically sound equivalent to the continuous total variation model. As a consequence, the labeling problem on the weighted graph can be solved efficiently using a recent algorithm for continuous convex optimization [10]. While ensuring connectivity constraints in image segmentation algorithms is usually difficult and in general not optimally solvable our method allows to include the connectivity prior directly in the convex optimization framework.

\subsection{Gradient and divergence operators on weighted graphs}

Let $G=(V, E, W)$ be a graph with the set of vertices $V$ with $|V|=n$, a set of edges $E \subset V \times V$ and a positive $n \times n$ weight matrix $W$ that assigns a weight to every edge of the graph. In the following we will use the shortest path tree computed by Dijkstra's algorithm as explained in the previous section as the underlying graph structure. This graph is a directed tree, therefore $W$ is not symmetric, as for an undirected graph, and an edge $e_{i j}=(i, j)$ is a directed edge from $i$ to $j$ with the positive weight $w_{i j}$. Furthermore the tree structure implies that $\sum_{i} \delta_{>0}\left(w_{i j}\right)=1 \forall j$ where $\delta_{>0}$ is the indicator function of values strictly greater zero.

We define the gradient and divergence operators on a discrete graph following [20] and [7, 15]. Let $f: V \rightarrow \mathbb{R}$ be a function of $\mathcal{H}(V)$, the Hilbert space of real-valued functions on the vertices of $G$ that is equipped with the inner product $\langle f, g\rangle_{\mathcal{H}(V)}=\sum_{v \in V} f(v) g(v)$. We define the difference operator $d: \mathcal{H}(V) \rightarrow \mathcal{H}(E)$ of $f$ on an edge $(i, j) \in E$ as

$$
(d f)\left(e_{i j}\right)=\sqrt{w_{i j}}(f(j)-f(i))
$$

This difference operator can be interpreted as the directional derivative $\delta_{i} f_{j}:=(d f)\left(e_{i j}\right)$ of a function $f$ at a vertex $i$ along the edge to vertex $j$.

We define the weighted gradient operator as the vector operator $\nabla_{i} f=\left(\delta_{i} f_{j}:(i, j) \in E\right)^{T}$. The $L^{2}$ norm of this vector is the local variation of $f$ at $v$

$$
\begin{aligned}
\left|\nabla_{i} f\right| & :=\sqrt{\sum_{(i j) \in E}\left(\delta_{i} f_{j}\right)^{2}} \\
& =\sqrt{\sum_{(i j) \in E} w_{i j}\left(f_{j}-f_{i}\right)^{2}}
\end{aligned}
$$

Equivalently, let $p: E \rightarrow \mathbb{R}$ be a function of $\mathcal{H}(E)$, the Hilbert space of real-valued functions on the edges of $G$, that is equipped with the inner product $\langle f, g\rangle_{\mathcal{H}(E)}=$ $\sum_{(i, j) \in E} f(i, j) g(i, j)$. The adjoint $d^{*}: \mathcal{H}(E) \rightarrow \mathcal{H}(V)$ of the difference operator is given by

$$
\langle d f, p\rangle_{\mathcal{H}(E)}=\left\langle f, d^{*} p\right\rangle_{\mathcal{H}(V)}
$$

Following the definitions of the inner products, the $d i$ vergence operator of $p$ at a node $i$ is

$$
\begin{aligned}
\operatorname{div} p_{i} & =-d^{*}(p)_{i} \\
& =\sum_{(j i) \in E} \sqrt{w_{j i}} p_{j i}-\sum_{(i j) \in E} \sqrt{w_{i j}} p_{i j}
\end{aligned}
$$

\subsection{The segmentation model in the weighted graph framework}

In this section we derive our image segmentation algorithm in the weighted graph framework. The relaxed labeling function $u$ assigns a value to every vertex, thus $u: V \rightarrow[0,1]$ is a function of $\mathcal{H}(V)$. Furthermore we are given a function $f: V \rightarrow \mathbb{R}$ that is defined by the conditional probabilities for foreground and background at every node as $f_{i}=-\log P_{\mathcal{F}}\left(x_{i}\right)+\log P_{\mathcal{B}}\left(x_{i}\right)$. The dual function $p: E \rightarrow \mathbb{R}$ is defined over the edges of the graph and belongs to $\mathcal{H}(E)$.

Given above definitions, we are able to formulate our segmentation model with local variation regularization on a weighted graph

$$
\min _{u \in[0,1]} \sum_{i \in V} f_{i} u_{i}+\lambda\left|\nabla_{i} u\right| .
$$

By comparing this term with the definition of the local variation Eq. 10 we observe that the weight of the regularizer $\lambda \in \mathbb{R}^{+}$corresponds to the edge weights $w_{i j}=\lambda^{2}$.

\subsection{Including the Connectivity Constraint}

Modeling the image segmentation model on a treestructured graph has two benefits. First, by defining the local variation regularization prior along the edges of the graph we are able to model anisotropic smoothness in direction of the elongated structure, similar to the method of [4] for tubular structure segmentation. The second advantage of our framework is that a connectivity constraint can be included very easily. Other researchers have already proposed such connectivity constraints, as they allow richer input to the algorithm especially in a user interactive setting [30]. While it is numerically expensive to include these constraints in graph cut frameworks [30] we will see in the following that they can be integrated into our framework very efficiently.

The segmentation model of Eq. 14 is extended with the connectivity constraint $\mathrm{C} 1$ which ensures that for every pixel labeled as foreground in the image there exists a connected path of foreground pixels to the root node. This is equivalent to the constraint that the label $u_{i}$ of a node $i$ is always greater or equal than the label of its neighbors with a larger distance to the root node: $d(i)<d(j) \Rightarrow u_{i} \geq u_{j}$. Because the graph structure is a shortest path tree, the condition $d(i)<d(j)$ is satisfied for all nodes $i$ and their child 
nodes $j$. The constraint $u_{i} \geq u_{j}$ then implies $\nabla_{i} u \leq 0 \forall i \in$ $V$. This constraint is linear in $u$, which preserves convexity of Eq. 14 and allows for an optimal solution.

When the object of interest is not a tree of thin elongated structures, for example in interactive image segmentation as depicted in Fig. 5, the local variation regularizer on the tree structure might lead to inappropriate results. In this case, we instead define the regularizer on the image grid which results in the standard total variation regularizer, that measures the boundary length in Euclidian space. While the connectivity constraint is still defined on the shortest path tree, the boundary length is measured on the 4-connected graph of the image grid.

\subsection{A primal-dual method for graph node labeling}

The definition of the weighted gradient and weighted divergence operators allows to formulate Eq. 14 as saddlepoint problem on a weighted graph

$$
\min _{u \in[0,1]} \max _{|p| \leq 1} \sum_{i \in V} f_{i} u_{i}+\left\langle\nabla_{i} u, p\right\rangle_{\mathcal{H}(E)} .
$$

The primal-dual formulation follows from the definition of the adjoint operator

$$
\min _{u \in[0,1]} \max _{|p| \leq 1} \sum_{i \in V} f_{i} u_{i}+\left\langle u_{i}, \operatorname{div} p_{i}\right\rangle_{\mathcal{H}(V)} .
$$

The update equations for the segmentation problem on a weighted graph can be derived similar to $[24,10]$. For the basic segmentation model without connectivity constraint they are equivalent to the continuous model, but in addition consistent with the different domains $E$ and $V$ :

$$
\begin{aligned}
u_{i}^{k+1} & =u_{i}^{k}+\tau \operatorname{div} p_{i}-\tau f \\
p_{i j}^{k+1} & =\pi_{|\cdot| \leq 1}\left(p_{i j}^{k}+\sigma \nabla_{i} \bar{u}^{k}\right) \\
\bar{u}_{i}{ }^{k+1} & =u_{i}^{k+1}+\theta\left(u_{i}^{k+1}-u_{i}^{k}\right)
\end{aligned}
$$

The parameter $\theta \in[0,1]$ controls the amount of overrelaxation. The primal and dual step sizes $\tau, \sigma>0$ need to be chosen according to $\tau \sigma L^{2}<1$, where $L=\left\|\nabla_{i}\right\|$ is the operator norm of the local variation operator [10].

Projections from one Hilbert space to the other are achieved by the corresponding operators div and $\nabla_{i}$. The projection $\pi_{|\cdot| \leq 1}(\cdot)$ computed by

$$
\pi_{|\cdot| \leq 1}\left(p_{i j}\right)=\frac{p_{i j}}{\max \left(\left|p_{i}\right|, 1\right)}
$$

projects the values of $p_{i j}$ to the unit ball, where

$$
\left|p_{i}\right|=\sqrt{\sum_{(i j) \in E} w_{i j} p_{i j}^{2}}
$$

is the vector norm $\left|p_{i}\right|$ over the edges incident to vertex $i$.

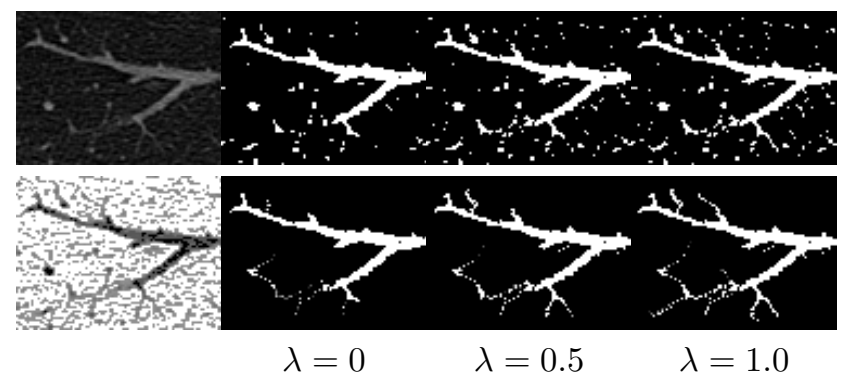

Figure 3: The connectivity prior allows to reconstruct a connected structure with superior suppression of noise compared to the same segmentation method without the connectivity prior. Different values of the regularizer weight $\lambda$ allow different levels of noise suppression. First column: a noisy input image and the data term. Upper row: without connectivity prior. Lower row: with connectivity prior.

The connectivity constraint is included by adding the indicator function

$$
\delta_{\leq 0}\left(\nabla_{i} u\right)= \begin{cases}0 & \text { if } \nabla_{i} u \leq 0 \\ \infty & \text { if } \nabla_{i} u>0\end{cases}
$$

to the segmentation model

$$
\min _{u \in[0,1]} \sum_{i \in V} f_{i} u_{i}+\lambda\left|\nabla_{i} u\right|+\delta_{\leq 0}\left(\nabla_{i} u\right) .
$$

The resulting optimization problem of Eq. 23 can be formulated in the primal-dual framework via the LegendreFenchel transform of the indicator function:

$$
\delta_{\leq 0}^{*}\left(\nabla_{i} u\right)=\sup _{\alpha \geq 0}\left\langle\nabla_{i} u, \alpha\right\rangle_{\mathcal{H}(E)} .
$$

Thus optimizing the image segmentation model with the connectivity constraint is equivalent to solving the saddlepoint-problem

$$
\min _{u \in[0,1]} \max _{\substack{|p| \leq 1 \\ \alpha \geq 0}} \sum_{i \in V} f_{i} u_{i}+\left\langle\nabla_{i} u, p\right\rangle_{\mathcal{H}(E)}+\left\langle\nabla_{i} u, \alpha\right\rangle_{\mathcal{H}(E)} .
$$

Finally, the update equations with connectivity constraint are

$$
\begin{aligned}
u_{i}^{k+1} & =u_{i}^{k}+\tau \operatorname{div} p_{i}+\tau \operatorname{div} \alpha_{i}-\tau f \\
p_{i j}^{k+1} & =\pi_{|\cdot| \leq 1}\left(p_{i j}^{k}+\sigma \nabla_{i} \bar{u}^{k}\right) \\
\alpha_{i j}^{k+1} & =\pi_{\geq 0}\left(\alpha_{i j}^{k}+\mu \nabla_{i} \bar{u}^{k}\right) \\
\bar{u}_{i}{ }^{k+1} & =u_{i}^{k+1}+\theta\left(u_{i}^{k+1}-u_{i}^{k}\right) .
\end{aligned}
$$

where the values of $\alpha_{i j}$ are defined over the edges of the graph and are projected to positive values by $\pi_{\geq 0}$. 


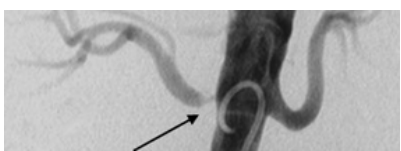

Input image

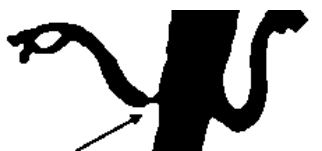

Result from [14]

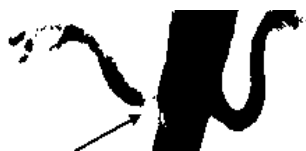

Without connectivity prior

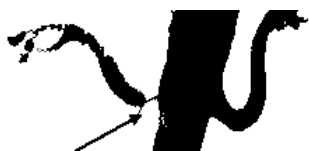

With connectivity prior

Figure 4: Results on two-dimensional medical image data. For comparison we show the results on images from [14]. First column: input image. Second column: Segmentation results from [14]. Third column: Segmentation without connectivity prior. Fourth column: Segmentation result with the proposed method. The connectivity prior enables to connect sparse foreground regions.
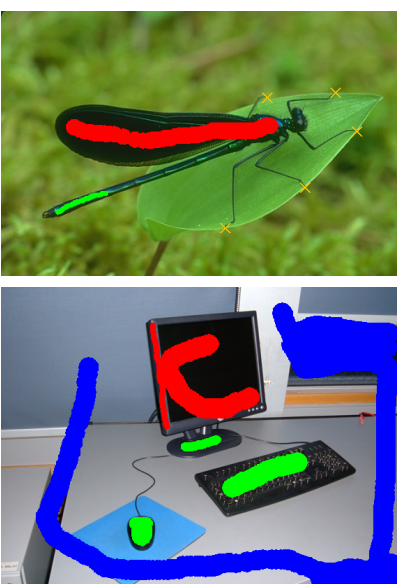

Input image
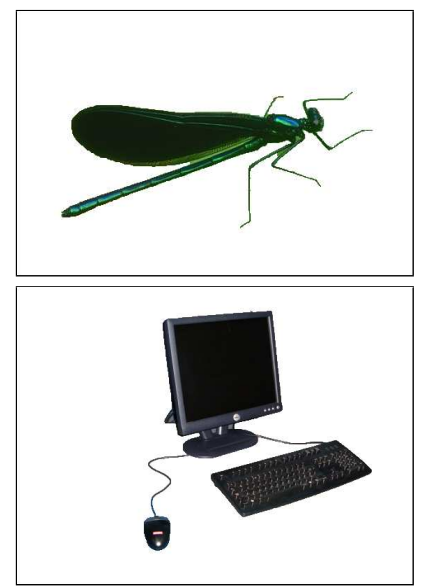

Results from [30]
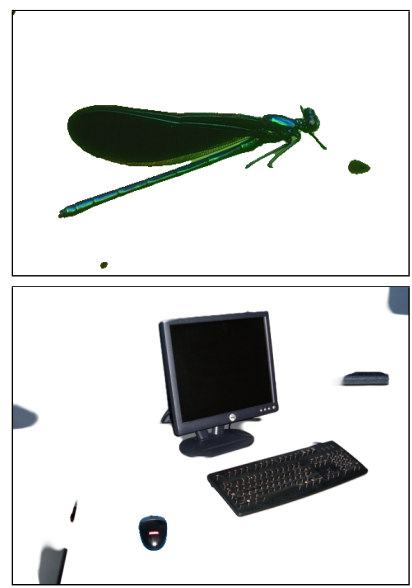

Without connectivity prior
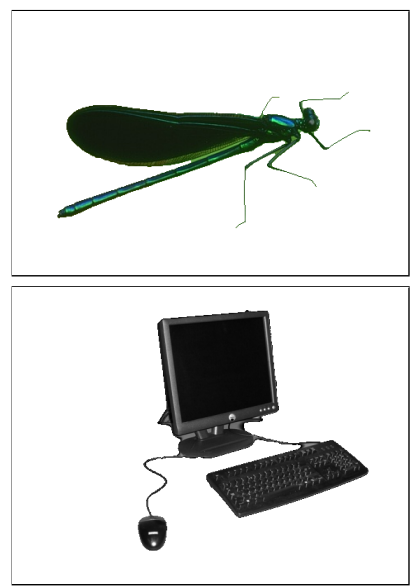

With connectivity prior

Figure 5: Results for user interactive segmentation. For comparison we show the results on an image from [30]. First column: Input image with user scribbles. The red scribble is the source foreground region of the geodesic shortest path tree, green scribbles are foreground regions that should be connected and blue scribbles are background regions. Second column: Segmentation results from [30]. Third column: Segmentation without connectivity prior. Fourth column: Segmentation result with the proposed connectivity prior.

\section{Experimental Results}

We applied our method to different types of image data. Figure 1 shows the result of our segmentation algorithm for the task of blood vessel segmentation in three dimensional CT angiography data ${ }^{1}$. When using our tree shape prior, even the small distal tips of the blood vessels are preserved in the final segmentation, while image noise that does not belong to the connected foreground region is successfully suppressed. For segmenting the whole blood vessel tree, only a single root node needs to be selected and the result is very robust to changes in this selection. To segment the volume of size $512 \times 512 \times 355$ voxels our algorithm needs 330 seconds on a single threaded 2.27 GHZ Intel Xeon architecture, which is less than 1 second per $512 \times 512$ volume slice. Another example, that demonstrates the advantage of the connectivity prior is shown in Figure 3. The connectivity prior clearly improves the segmentation result especially

\footnotetext{
${ }^{1}$ CT dataset taken from the Vessel Segmentation in the Lung 2012 Grand Challenge http://vessel12.grand-challenge.org.
}

for noisy input data. Figure 4 shows additional results of our tree shape prior on two dimensional medical image data.

Furthermore, the connectivity prior is also a useful extension in an interactive segmentation framework. Figure 5 shows an input image with additional user scribbles, that provide hard constraints for foreground and background regions. With these scribbles the user can describe how the shortest path tree is constructed. One foreground region acts as the root node of the shortest path tree. Additional foreground regions can be added via brush strokes that should be connected to the root region. Given the user scribbles the probability density functions for foreground and background are estimated with a Parzen window estimator, with a kernel function, for example a Gaussian kernel $k_{\sigma}\left(I(x)-I_{s}\right)$, centered at every image value $I_{s}$ of the user scribbles.

The method was quantitatively evaluated on the DRIVE database [27] of digital retinal images for vessel extraction. Because the main contribution in this work is the connectivity prior and not the design of a special data term for retinal 


\begin{tabular}{llll}
\hline \hline & Accuracy & Sensitivity & Specificity \\
\hline $2^{\text {nd }}$ Observer & $94,73 \%$ & & \\
\hline Connectivity Prior & & & \\
w Bending Energy & $94,57 \%$ & $84,54 \%$ & $95,83 \%$ \\
w/o Bending Energy & $94,56 \%$ & $84,69 \%$ & $95,79 \%$ \\
\hline Staal & $94,42 \%$ & & \\
\hline \hline
\end{tabular}

Figure 6: Quantitative evaluation results of the proposed method on the DRIVE database [27]. A combination of the connectivity prior with the method of Staal leads to the most accurate method on this dataset, almost reaching the performance of a second human observer.

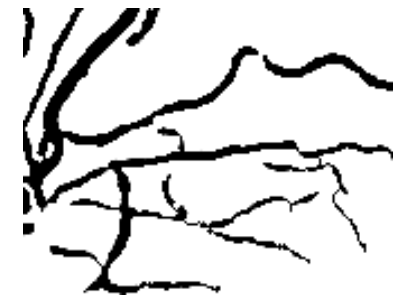

Without bending energy

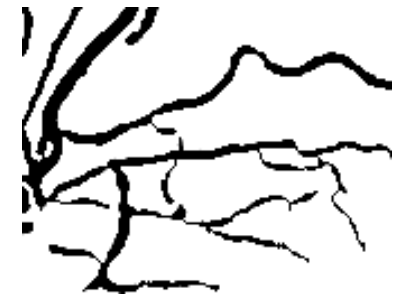

With bending energy
Figure 8: Magnified result from the DRIVE dataset. The bending energy term changes the topology of the connected structure.

blood vessel detection, the performance of the tree shape prior was evaluated by using the method of Staal [27] as data term. This is the currently best performing method in the benchmark with an accuracy of $94,42 \%$. By combining this method with the proposed connectivity prior the accuracy can be increased to $94,57 \%$. Therefore this is the highest accuracy reported for this database and we were able to reduce the distance to the human observer $(94,73 \%)$ by a factor of two. Fig. 7 shows the segmentation results on one of the images in the dataset.

Including the bending energy term leads to an increased specificity and a slightly increased accuracy, while the sensitivity is decreased. Overall, the number of true positive classified pixels is increased and the number of true negatives is slightly decreased. The topological difference of the segmentation result is depicted in Fig. 8.

\section{Conclusion}

In this work we presented a novel method for image segmentation with connectivity constraints. While solving the image segmentation problem with general connectivity constraints is NP-hard, we propose to formulate the constraint on a shortest geodesic path tree, leading to the novel tree shape prior.

We show that our method can be successfully applied to medical image segmentation problems in angiography and retinal blood vessel extraction, where thin structures otherwise would not be preserved by boundary length regularizers. Experiments on a public dataset show that combining the connectivity prior with existing image segmentation methods clearly improves the performance.

To solve the optimization problem, we generalized an efficient primal dual optimization algorithm for arbitrary graphs. Future work will focus on utilizing the iterative structure of the algorithm for a parallelized implementation on the GPU.

\section{Acknowledgements}

This research was supported by the ERC Starting Grant "ConvexVision" and the Technische Universität München Institute for Advanced Study, funded by the German Excellence Initiative.

\section{References}

[1] A. A. Amini, T. E. Weymouth, and R. C. Jain. Using dynamic programming for solving variational problems in vision. Pattern Analysis and Machine Intelligence, IEEE Transactions on, 12(9):855-867, 1990. 3

[2] X. Bai and G. Sapiro. A geodesic framework for fast interactive image and video segmentation and matting. In Computer Vision, 2007. ICCV 2007. IEEE 11th International Conference on, pages 1-8. IEEE, 2007. 2

[3] C. Bauer, T. Pock, E. Sorantin, H. Bischof, and R. Beichel. Segmentation of interwoven 3d tubular tree structures utilizing shape priors and graph cuts. Medical image analysis, 14(2):172-184, 2010. 2

[4] F. Benmansour and L. Cohen. Tubular structure segmentation based on minimal path method and anisotropic enhancement. International Journal of Computer Vision, 92:192210, 2011. 2, 4

[5] F. Benmansour, L. D. Cohen, M. Law, and A. Chung. Tubular anisotropy for $2 \mathrm{~d}$ vessel segmentation. In Computer Vision and Pattern Recognition, 2009. CVPR 2009. IEEE Conference on, pages 2286-2293. IEEE, 2009. 2

[6] M. Bergou, M. Wardetzky, S. Robinson, B. Audoly, and E. Grinspun. Discrete Elastic Rods. ACM Transactions on Graphics (SIGGRAPH), 27(3):63:1-63:12, aug 2008. 3

[7] S. Bougleux, A. Elmoataz, and M. Melkemi. Discrete regularization on weighted graphs for image and mesh filtering. In F. Sgallari, A. Murli, and N. Paragios, editors, SSVM, volume 4485 of Lecture Notes in Computer Science, pages 128139. Springer, 2007. 4

[8] Y. Boykov and V. Kolmogorov. An experimental comparison of min-cut/max- flow algorithms for energy minimization in vision. Pattern Analysis and Machine Intelligence, IEEE Transactions on, 26(9):1124 -1137, sept. 2004. 1

[9] Y. Boykov, O. Veksler, and R. Zabih. Fast approximate energy minimization via graph cuts. Pattern Analysis and Machine Intelligence, IEEE Transactions on, 23(11):12221239, 2001. 1, 2 


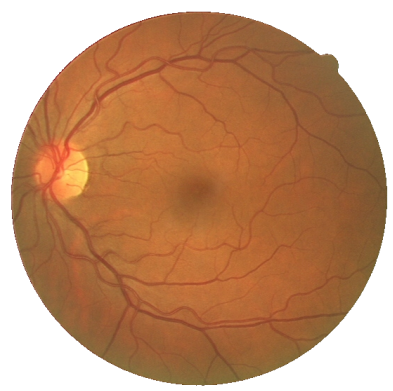

Input image

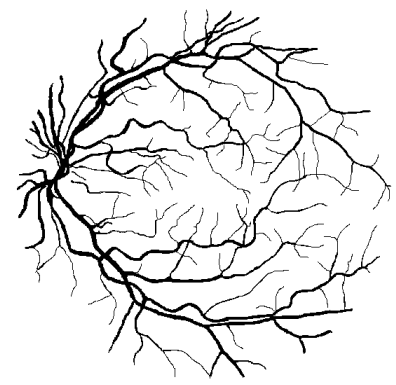

Ground truth

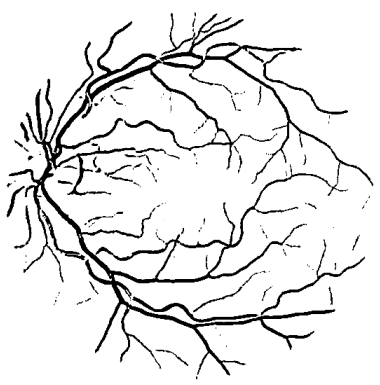

Result from [27]

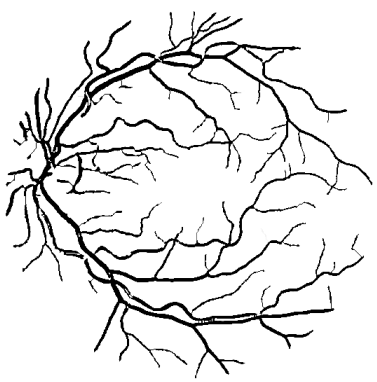

With connectivity prior

Figure 7: Results on an image of the DRIVE dataset. The connectivity prior increases the segmentation accuracy.

[10] A. Chambolle and T. Pock. A first-order primal-dual algorithm for convex problems with applications to imaging. $J$. Math. Imaging Vis., 40(1):120-145, May 2011. 4, 5

[11] C. Chen, D. Freedman, and C. H. Lampert. Enforcing topological constraints in random field image segmentation. In Computer Vision and Pattern Recognition (CVPR), 2011 IEEE Conference on, pages 2089-2096. IEEE, 2011. 1

[12] A. Criminisi, T. Sharp, and A. Blake. Geos: Geodesic image segmentation. In Computer Vision-ECCV 2008, pages 99112. Springer, 2008. 2

[13] E. Dijkstra. A note on two problems in connexion with graphs. Numerische Mathematik, 1:269-271, 1959. 3

[14] N. Y. El-Zehiry and L. Grady. Fast global optimization of curvature. In CVPR, pages 3257-3264. IEEE, 2010. 1, 6

[15] A. Elmoataz, O. Lezoray, and S. Bougleux. Nonlocal discrete regularization on weighted graphs: A framework for image and manifold processing. Image Processing, IEEE Transactions on, 17(7):1047 -1060, july 2008. 4

[16] A. F. Frangi, W. J. Niessen, K. L. Vincken, and M. A. Viergever. Multiscale vessel enhancement filtering. In Medical Image Computing and Computer-Assisted InterventationMICCAI98, pages 130-137. Springer, 1998. 2

[17] B. Goldluecke and D. Cremers. Introducing total curvature for image processing. In IEEE International Conference on Computer Vision (ICCV), 2011. 1

[18] L. Grady. Random walks for image segmentation. Pattern Analysis and Machine Intelligence, IEEE Transactions on, 28(11):1768 -1783, Nov. 2006. 1

[19] X. Han, C. Xu, and J. L. Prince. A topology preserving level set method for geometric deformable models. Pattern Analysis and Machine Intelligence, IEEE Transactions on, 25(6):755-768, 2003. 1

[20] M. Hein, J.-Y. Audibert, and U. von Luxburg. Graph laplacians and their convergence on random neighborhood graphs. Journal of Machine Learning Research, 8:13251368, 2007. 4

[21] K. Krissian, G. Malandain, N. Ayache, R. Vaillant, and Y. Trousset. Model-based detection of tubular structures in $3 \mathrm{~d}$ images. Computer vision and image understanding, 80(2):130-171, 2000. 2

[22] D. Lesage, E. D. Angelini, I. Bloch, and G. Funka-Lea. A review of $3 \mathrm{~d}$ vessel lumen segmentation techniques: Models, features and extraction schemes. Medical image analysis, 13(6):819-845, 2009. 2

[23] S. Nowozin and C. H. Lampert. Global connectivity potentials for random field models. In Computer Vision and Pattern Recognition, 2009. CVPR 2009. IEEE Conference on, pages 818-825. IEEE, 2009. 1, 2

[24] T. Pock, D. Cremers, H. Bischof, and A. Chambolle. An algorithm for minimizing the piecewise smooth mumford-shah functional. In IEEE International Conference on Computer Vision (ICCV), Kyoto, Japan, 2009. 5

[25] T. Schoenemann, F. Kahl, S. Masnou, and D. Cremers. A linear framework for region-based image segmentation and inpainting involving curvature penalization. International Journal of Computer Vision, 2012. to appear. 1

[26] J. A. Sethian. A fast marching level set method for monotonically advancing fronts. Proceedings of the National Academy of Sciences, 93(4):1591-1595, 1996. 3

[27] J. Staal, M. D. Abràmoff, M. Niemeijer, M. A. Viergever, and B. van Ginneken. Ridge-based vessel segmentation in color images of the retina. Medical Imaging, IEEE Transactions on, 23(4):501-509, 2004. 6, 7, 8

[28] J. Tsitsiklis. Efficient algorithms for globally optimal trajectories. Automatic Control, IEEE Transactions on, 40(9):1528 -1538 , sep 1995. 3

[29] M. Unger, T. Pock, W. Trobin, D. Cremers, and H. Bischof. Tvseg-interactive total variation based image segmentation. In British Machine Vision Conference, volume 2. Citeseer, 2008. 1

[30] S. Vicente, V. Kolmogorov, and C. Rother. Graph cut based image segmentation with connectivity priors. In CVPR. IEEE Computer Society, 2008. 1, 2, 4, 6

[31] Y. Zeng, D. Samaras, W. Chen, and Q. Peng. Topology cuts: A novel min-cut/max-flow algorithm for topology preserving segmentation in $\mathrm{n}-\mathrm{d}$ images. Computer vision and image understanding, 112(1):81-90, 2008. 1 